\title{
Exploring a Recommendation System of Free E-learning Platforms: Functional Architecture of the System
}

\author{
https://doi.org/10.3991/ijet.v12i02.6381 \\ Mohammed Ouadoud \\ Abdelmalek Essaadi University, Tetouan, Morocco \\ mohammed. ouadoud@gmail.com \\ Mohamed Yassin Chkouri \\ Abdelmalek Essaadi University, Tetouan, Morocco \\ yassin.chkouri@gmail.com \\ Amel Nejjari \\ Abdelmalek Essaadi University, Tetouan, Morocco \\ amelnejjari@yahoo.fr \\ Kamal Eddine El Kadiri \\ Abdelmalek Essaadi University, Tetouan, Morocco \\ kamalelkadiri@yahoo.fr
}

\begin{abstract}
This paper presents the functional architecture of a recommendation system of free e-learning platforms that we have implemented in order to facilitate the choice of the most suitable e-learning platform to meet the objectives, specifications and criteria chosen by the institution. Thus, any random choice entails a loss of money, effort and time loss, for porters and device designers, and this is for various reasons (cost, utility, usability, etc.). Notably, this system takes into account more than 20 platforms. The choice of these platforms is based on a methodical and systemic approach that identifies the adequate criteria to the objectives and specifications chosen by the institution, depending on the objects and pedagogical tools related to the recommended teaching and learning device, in order to retain the most suitable e-learning platform.

This paper is motivated by our will to clarify and support users in their choice of the most suitable platform to meet their needs and to benefit a maximum from the potential offered by technologies in pedagogy.
\end{abstract}

Keywords - Recommendation system, e-learning platform, LMS, functional architecture, LeaderTICE.

\section{Introduction}

E-learning is a fast and efficient way for providing and sharing knowledge with learners in different parts of the world. 
According to [6,7] , it is defined as the following: "E-learning uses the Internet or other digital content for learning and education activities, that takes full advantage of modern educational technology to provide a new mechanism for communication and learning environment rich in resources to achieve a new way of learning."

In the 20th century, there was an international movement in favor of e-learning integration in higher education. This movement has been operationalized due to the variety of the educational offer by universities, which most have opted to diversify knowledge dissemination means (sounds, images, animations ... etc.) to meet the needs of their target public. If access to knowledge was previously conditioned by the physical presence in the classroom, technology enables its learners to exceed this condition of presence and be opened towards other learning modalities today. We can say that e-learning brings solutions within the distance learning framework without for as many without pretending to represent the panacea for all pedagogical dysfunctions. Among these solutions, distance learning seems to be the challenge ahead to face the new training requirements in the digital era.

In the case of our study, the e-learning solutions that interest us are free e-learning platforms, because their costs, their states of development, their directions and used technologies rendered them very close to the axis of this study.

During the last decade, the e-learning platforms have evolved a lot. However, several comparative studies have been developed previously $[8,9,10,11,15,16,17,18$, $19]$, but they have been abandoned because their life cycle is changing apace. In such a context of proliferation of the training, the choice becomes difficult.

Consequently, any random choice causes a loss of money, effort and time.

Thereby, we developed a recommendation system of platforms, based on a comparative and analytical study of the free e-learning platforms [12], with a view to facilitate the choice of the most suitable e-learning platform according to the objectives and specifications of any institution, and this is based on an evaluation approach of the e-learning platforms quality [1].

For our study, in the "functional architecture of the system" section, we present the functional architecture of the recommendation system of used e-learning platforms. This section mainly consists of three subsections:

1. The formalization of the needs: the system will facilitate the selection of the elearning platform that allows the dissemination of trainings and will allow to choose the one that suits best your training ecosystem.

2. The choice of pedagogical tools: the system gives you the possibility to choose the characteristics and pedagogical tools adapted to your specifications, according to an evaluation approach of the e-learning platforms quality [1].

3. The analysis and results processing: the system does the analysis and treatment of the choices obtained, in order to recommend the most suitable platform to your institutional goals. 


\section{LeaderTICE Recommendation System}

LeaderTICE is a recommendation system of free e-learning platforms, under the GNU / GPL V3 [3], that we implemented [2] in order to facilitate the choice of elearning platforms, seeing that any random choice entails a money, effort and time loss to porters and devices designers, and this is for various reasons (cost, utility, usability ...). Notably, this system takes into account several platforms selected among 600 platforms listed by THOT CURSUS directory (e-learning platforms directory, LMS and LCMS platforms).

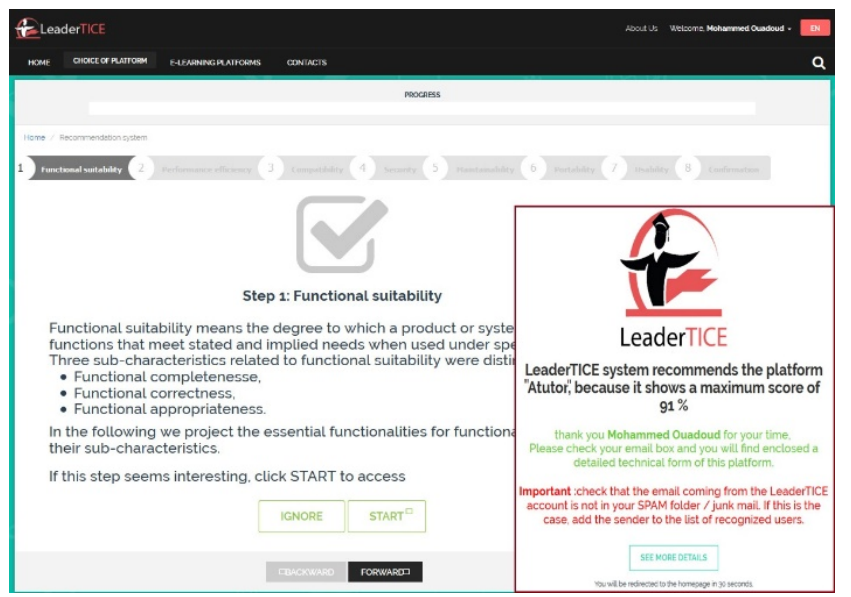

Fig. 1. LeaderTICE recommendation system of e-learning platforms

The LeaderTICE system is mainly based on our evaluation approach of the elearning platforms quality [1], [12]. Several platforms evaluation approaches were encountered in the literature $[13,14]$. However, they have not been adopted, because these studies are focusing on the functional aspect mainly, forgetting other very important aspects such as: security, maintainability, portability, compatibility, performance efficiency and usability. For this reason, the implementation of the system is based on a methodical and systemic approach $[1,12]$ taking into account the software engineering aspects, learning theories and current educational tools. Our main goal is to propose the most suitable platform which meshes with the objectives and contexts of teaching and learning of any institution.

\section{Functional Architecture of the System}

Figure 2 illustrates the general principle of the LeaderTICE system functioning by presenting the main functionalities associated with the main actors. This figure represents the articulation between learning objects and pedagogical tools that might underlie your pedagogical scenario when exploring the recommendation system (of elearning platforms) LeaderTICE. 


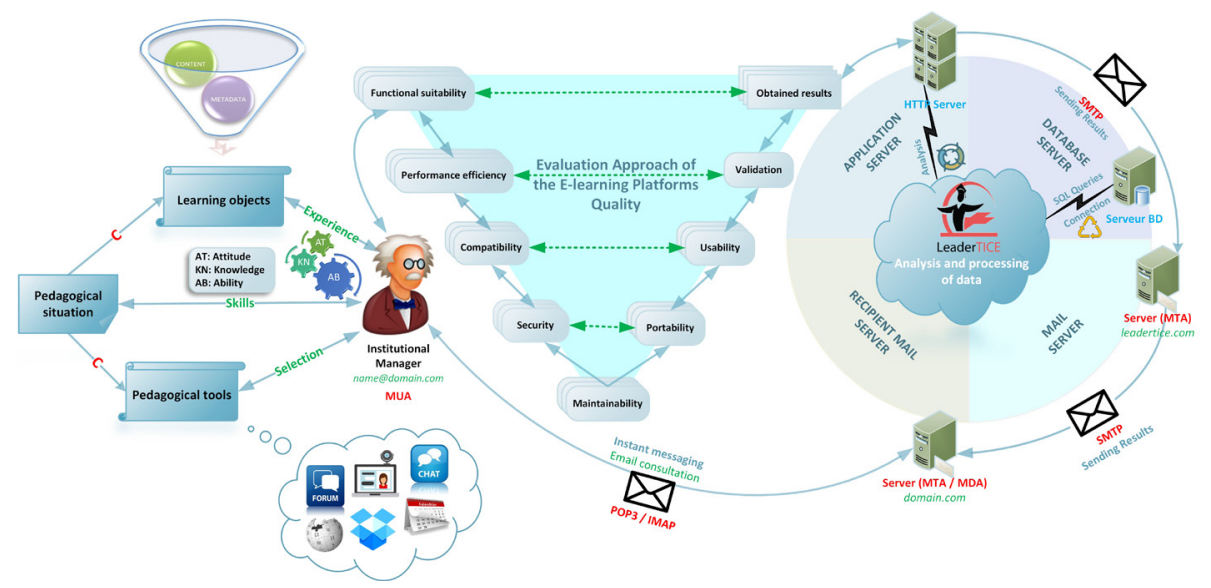

Fig. 2. Functional architecture of the LeaderTICE recommendation system of free e-learning platforms

LeaderTICE allows you choose the most suitable e-learning platform for your objects and your requested pedagogical tools. And this is according to three main steps have been distinguished according to the circuit shown in figure 2. These steps are: the formalization of needs, the choice of pedagogical tools, the results analysis and processing.

\subsection{Formalization of needs}

In the choice process, the formalization of needs will facilitate the selection of the e-learning platform that will allow the dissemination of trainings. To choose the one that will be best integrated with our training ecosystem, we, therefore, must arbitrate between:

- Pedagogical situation, which presents a genuine assumed, of simultaneous and complementary way, that a certain destabilization same time is a chosen fulcrum [5], after determining the objectives, specifying the pedagogical used method (expositive, interrogative, collaborative work ...) and the used supports (documents, video, image ...).

- Learning Objects, which present the smallest information units or smallest information processing tools (applications or tutorials) used in a teaching context with a pedagogical intention aiming at facilitating the learning through technological support [4].

- Functional or organizational pedagogical tools... such as forum, wiki, videoconferencing, activities, tests, certification... corresponding to your pedagogical expectations and your teaching / learning situation.

- Financial implications, any wrong choice of e-learning platform will have financial repercussions. 


\subsection{Choice of pedagogical tools}

After the formalization of the needs, the institutional manager has to choose the characteristics and pedagogical tools adapted to the institutional specificities, according to a questionnaire based on an evaluation approach of the e-learning platforms quality [1]. The latter presents an overview on the utility and usability dimensions with their adapted measures and criteria to evaluate e-learning platforms. This approach is mainly composed of seven characteristics:

- Functional suitability which means the degree to which a product or system provides functions that meet stated and implied needs when used under specified conditions.

- Performance efficiency which means the performance relative to the amount of resources used under stated conditions.

- Compatibility which signifies the degree to which a product, system or component can exchange information with other products, systems or components, and/or perform its required functions, while sharing the same hardware or software environment.

- Security which means the degree to which a product or system protects information and data so persons or other products or systems have the degree of data access appropriate to their types and levels of authorization.

- Maintainability. It means the degree of effectiveness and efficiency with which a product or system can be modified by the intended maintainers.

- Portability which signifies the degree of effectiveness and efficiency with which a system, product or component can be transferred from one hardware, software or other operational or usage environment to another.

- Usability refers to the degree to which a product or system can be used by specified users to achieve specified goals with effectiveness, efficiency and satisfaction in a specified context of use.

Thus, it should be mentioned that you have the right to choose just the adapted characteristics and sub-characteristics to your institutional needs, your technical imperatives and your functional and organizational expectations.

Finally, before having validated your choices, you can do a general verification of your chosen characteristics by pressing the backward button.

\subsection{The results analysis and processing}

After validating your choices, the LeaderTICE system does the analysis and processing of results obtained by algorithmic programs interpreted in PHP language via an "Apache HTTP ${ }^{1}$ Server" application server, who took charge of the PHP language interpretation, the analysis and processing of the code, and interconnection to the MySQL database. In order to display the treatment result and recommend the most suitable platform to your institutional goals, according to the criteria and specifica-

${ }^{1}$ HTTP : Hypertext Transfer Protocol 


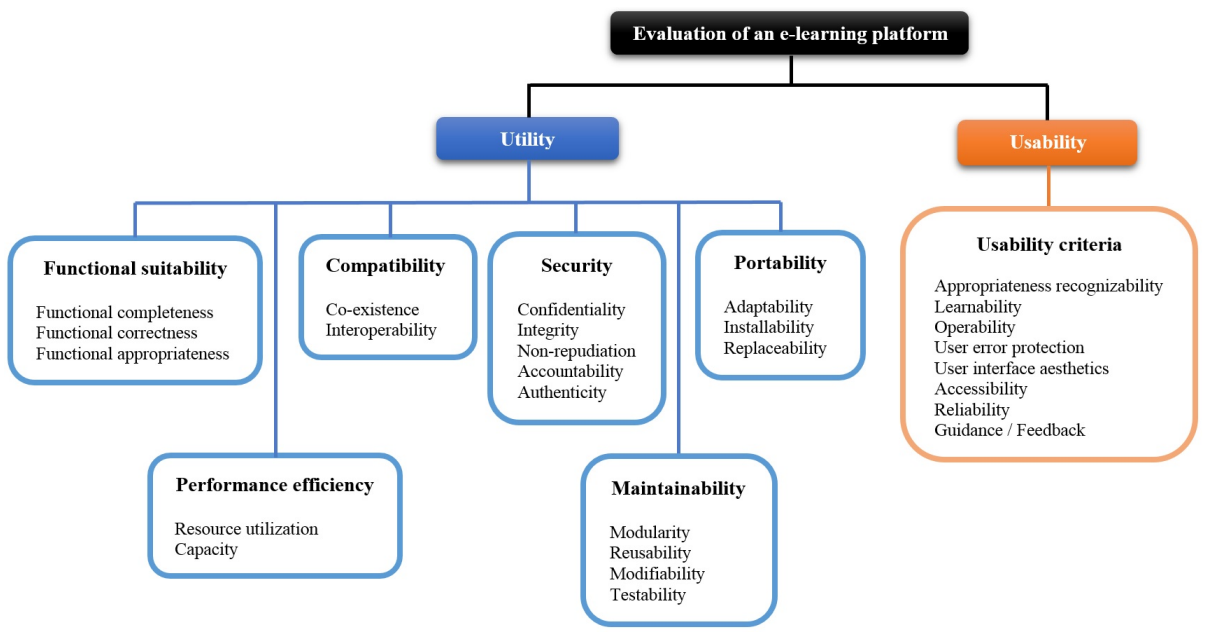

Fig. 3. Quality model selected for choosing an e-learning platform [1]

tions that you have expressed. Thereafter, the "Apache HTTP Server" application server sends the results obtained in the previous step by mailing way via the SMTP ${ }^{2}$ communication protocol, used to transfer the electronic mails towards the mail server. Furthermore, the $\mathrm{MTA}^{3}$ mail server responsible for transport, by its turn, returns the mail of received results by the application server "Apache HTTP Server" by mailing way via the SMTP communication protocol toward the MTA recipient's mail server. Lastly, the latter delivers, then, the mail that contains the result obtained in the incoming electronic mail server (named MDA for Mail Delivery Agent), which then stores the mail in the meantime, via the POP3 / IMAP protocol which allows you to retrieve the electronic mail containing the result.

\section{Conclusion and Perspectives}

The subject of this paper is very important because the free platforms are, on the one hand, an actuality domain in Software Engineering and in Pedagogical Engineering Multimedia. On the other hand, the application of these platforms in e-learning reaches a vast clientele.

A platform choice, pledge of success and sustainability for pedagogical device that also seems in adequacy with the free platforms' philosophy and their community dynamic. Furthermore, academically, it seemed interesting to apply for the first time a recommendation system of the free e-learning platforms based on a methodical and systemic approach inspired by the software engineering to facilitate the choice of the most suitable e-learning platform to the objectives and institutional specifications.

\footnotetext{
${ }^{2}$ SMTP : Simple Mail Transfer Protocol

${ }^{3}$ MTA : Mail Transfer Agent
} 
In a later phase, two important functionalities will be added to the system to facilitate recommending e-learning platforms, namely:

- Implementation System. The system will offer the possibility to automatically install option the recommended platform.

- Personalization system. The recommended platform will be installed in a personalized manner according to the needs you have expressed.

In this perspective, an experiment is therefore indispensable to validate what has been developed in the context of this paper. Indeed, the latter will be based on the results of the establishing of a teaching / learning device in Moroccan university, based on a platform known as intelligent and anthropocentric relying on a reduced learning design, with a view to enhance distance learning.

\section{$5 \quad$ References}

[1] M. Ouadoud, MY. Chkouri, A. Nejjari, KE. EL Kadiri, (2016) Studying and Analyzing the Evaluation Dimensions of E-learning Platforms Relying on a Software Engineering Approach. International Journal of Emerging Technologies in Learning (iJET). 2016, Vol. 11 Issue 1, p11-20. 10p. https://doi.org/10.3991/ijet.v11i01.4924

[2] M. Ouadoud, (2016) "LeaderTICE - Choosing e-learning platform." [Online]. Available: http://leadertice.com/ChoixDePlateforme. [Accessed: 03-Mar-2016].

[3] M. Ouadoud, (2016) "LeaderTICE - Our use terms and conditions." [Online]. Available: http://leadertice.com/TermesEtConditions. [Accessed: 03-Mar-2016].

[4] CREPUQ - Novasys, 2003 ;p. 133

[5] Astolfi, J.- P. (1992). L'école pour apprendre. Paris, ESF

[6] Liu, C.-H.,"The comparison of learning effectiveness between traditional face-to-face learning and e-learning among goal-oriented users", in Digital Content, Multimedia Technology and its Applications (IDC), 2010 6th International Conference on. 2010. IEEE.

[7] Faten Karim, Dr. Robert Goodwin, « Using Cloud Computing in Elearning Systems », International Journal of Advanced Research in Computer Science \& Technology (IJARCST), Vol. 1 Issue 1 Oct-Dec 2013,pp.66-67.

[8] B. Dimet, "Etude comparative technique et pédagogique des plates-formes pour la formation ouverte et à distance," 15-Jan-2006. [Online]. Available: http://www.portices.fr/for mation/Res/-Formation/Plateformes/Pf1Resume.html. [Accessed: 06-Jun-2016].

[9] S. Menasri, "Etude comparative de plateformes d'enseignement en ligne (e-learning) utilisées dans un contexte universitaire,” Jun-2004. [Online]. Available: http://urfist.enc.sorbon ne.fr/anciensite/archives/gremi/gremi10juin04/comparatif.htm\#. [Accessed: 06-Jun-2016].

[10] DOGBE-SEMANOU, DA. Koffi, A. DURAND, M. LEPROUST, H. VANDERSTICHEL, Etude comparative de plates-formes de formation à distance. le cadre du Projet@2L Octobre, 2007. https://www.projet-plume.org/files/Choix plateforme a21.pdf.

[11] M. Kaddouri and A. Bouamri, "Usage de plateformes d'enseignement à distance dans l'enseignement supérieur marocain: avantages pédagogiques et difficultés d'appropriation," Questions Vives. Recherches en éducation, no. Vol.7 n¹4, pp. 107-118, Dec. 2010.

[12] M. Ouadoud, M. Y. Chkouri, A. Nejjari, and K. E. E. Kadiri, "Studying and comparing the free e-learning platforms," in 2016 4th IEEE International Colloquium on Information Sci- 
Paper-Exploring a Recommendation System of Free E-learning Platforms: Functional Architecture of the System

ence and Technology (CiSt), 2016, pp. 581-586. https://doi.org/10.1109/CIST.2016. 7804953

[13] A. Lablidi, A. Abourrich and M. Talbi, (dec-2009) « Démarche préconisée pour évaluer une plate-forme », Association EPI, [Online]. Available: www.epi.asso.fr/revue/articles/ a0912b.htm [Accessed: 06-Jun-2016].

[14] Aska, Le Préau \& Klr.fr (2000). « Choisir une solution de téléformation : 2000 study : l'offre de plates-formes et de portails de téléformation » / [Study conducted by A. Bouthry, P. Chevalier, S. Ravet, and al.].

[15] N. EL MAWAS, L. Oubahssi, and P. Laforcade, (10-Jul-2014) "Étude comparative de plateformes de formation à distance," GRAPHIT-D2.5\&2.2. [Online]. Available: http://wwwlium.univ-lemans.fr/ laforcad/graphit/wp-content/uploads/2014/08/GRAPHIT-D2.52.2V6.pdf. [Accessed: 06-Jun-2016].

[16] l'OVAREP (Observatoire des ressources multimédias), 2000 Étude comparative technique et pédagogique des plateformes pour la formation ouverte et à distance

[17] S. Graf and B. List, "An evaluation of open source e-learning platforms stressing adaptation issues," in Fifth IEEE International Conference on Advanced Learning Technologies (ICALT'05), 2005, pp. 163-165. https://doi.org/10.1109/ICALT.2005.54

[18] S. Galloy, C. Haas, M. Lodewijck, (Mai-2002), “Analyse d'environnements de formation à distance, " CIFoP - Le Centre Interuniversitaire de Formation Permanente. [Online]. Accessed: http://www.formahetice.ulg.ac.be/upload/wiki/b/b5/Document2.pdf. [Accessed: 12-juill-2016].

[19] S. Caro Dambreville, "Evaluer un dispositif de formation à distance Principes et retour d'expérience," Revue des Interactions Humaines Médiatisées. Vol 9 º2, 2008. [Online]. Accessed: http://europia.org/RIHM/V9N2/RIHM9(2)-3CaroDemberville.pdf. [Accessed: 12-juill-2016].

\section{Authors}

M. Ouadoud is with Laboratory of Informatics, Research Operational and Statistic Applied (LIROSA) at Faculty of Sciences, Abdelmalek Essaadi University, Tetouan, Morocco. (E-mail: mohammed.ouadoud@gmail.com).

M. Y. Chkouri is with Laboratory of the Information System and Software Engineering (SIGL) at National School of Applied Sciences, Abdelmalek Essaadi University, Tetouan, Morocco. (E-mail: yassin.chkouri@gmail.com).

A. Nejjari is with Laboratory of the Information System and Software Engineering (SIGL) at National School of Applied Sciences, Abdelmalek Essaadi University, Tetouan, Morocco. (E-mail: amelnejjari@yahoo.fr).

K. E. El Kadiri is with Laboratory of Informatics, Research Operational and Statistic Applied (LIROSA) at Faculty of Sciences, Abdelmalek Essaadi University, Tetouan, Morocco. (E-mail: kamalelkadiri@yahoo.fr).

Submitted, 31 October 2016. Published as resubmitted by the authors on 11 November 2016 